Pacific Journal of Mathematics

PERIODIC JACOBI-PERRON ALGORITHMS AND 


\title{
PERIODIC JACOBI-PERRON ALGORITHMS AND FUNDAMENTAL UNITS
}

\author{
NAMBury S. RAJU
}

In this paper the author states a class of infinitely many real cubic fields for which the Jacobi-Perron algorithm of a properly chosen vector becomes periodic and calculates explicitly a fundamental unit for each field. The main results of this paper are: Let $m=a^{6}+3 a^{3}+3, \quad \omega=\sqrt[3]{m}, m$ cube free $a \in N$; then the Jacobi-Perron algorithm of $a^{(0)}=\left(\omega, \omega^{2}\right)$ is periodic. The length of the primitive preperiod is four and the length of the primitive period is three. A fundamental unit in $Q(\omega)$ is given by $e=a^{3}+1-a \omega$.

1. Introduction. The Jacobi algorithm [9] which was generalized by Perron [11] for any dimension $n \geqq 3$ proceeds as follows. Let $a^{(0)}$ be a vector in $R_{n-1}$; then the sequence $\left\langle a^{(v)}\right\rangle$ is called the Jacobi-Perron algorithm, if, for $a^{(v)}=\left(a_{1}^{(v)}, \cdots, a_{n-1}^{(v)}\right),(v=0,1, \cdots)$

$$
\begin{aligned}
& a^{(v+1)}=\frac{1}{a_{1}^{(v)}-b_{1}^{(v)}}\left(a_{2}^{(v)}-b_{2}^{(v)}, \cdots, a_{n-1}^{(v)}-b_{n-1}^{(v)}\right), \quad\left(b_{1}^{(v)} \neq a_{1}^{(v)} ; v=0,1, \cdots\right) \\
& b_{\imath}^{(v)}=\left[a_{\imath}^{(v)}\right], \quad(i=1, \cdots, n-1 ; V=0,1, \cdots) .
\end{aligned}
$$

For notation see Bernstein's book [7, pp. 11-18].

The Jacobi-Perron algorithm of a vector $Q^{(0)} \in R_{n-1}$ is called periodic, if there exist two rational integers $L$ and $M, L \geqq 0, M \geqq 1$, such that

$$
a^{(M+V)}=a^{(V)}, \quad(V=L, L+1, \cdots) .
$$

If $\min L=l, \min M=m$, then the sequence of vectors

$$
a^{(0)}, a^{(1)}, \cdots, a^{(L-1)}
$$

is called the primitive preperiod of the Jacobi-Perron algorithm, and the sequence of vectors

$$
a^{(L)}, a^{(L+1)}, \cdots, a^{(L+M-1)}
$$

is called primitive period. The $l$ and $m$ are called respectively the lengths of the primitive preperiod and period. If $l=0$, the algorithm is said to be purely periodic. By definition, from any periodic Jacobi- 
Perron algorithm a purely periodic one can be derived. Perron [11] proved that if the Jacobi-Perron algorithm of $a^{(0)}$ is periodic, then all the components of $a^{(v)}(v=0,1, \cdots)$ are algebraic numbers belonging to a field of degree $\leqq n$. In [1-5], Bernstein has stated a few classes of infinitely many real algebraic fields, for which the Jacobi-Perron algorithm of a properly chosen vector $a^{(0)}$ becomes periodic.

For later purposes, we need the following two important results about units in the field $Q\left(a_{1}^{(0)}, a_{2}^{(0)}, \cdots, a_{n-1}^{(0)}\right)$ :

THEOREM 1. (8). If the Jacobi-Perron algorithm of $a^{(0)}=$ $\left(a_{1}^{(0)}, \cdots, a_{n-1}^{(0)}\right)$ becomes periodic, with length lof the primitive preperiod and length $m$ of the primitive period, then

$$
e=\prod_{i=l}^{l+m-1} a_{n-1}^{(l)} \omega
$$

is a unit in $Q\left(a_{1}^{(0)}, \cdots, a_{n-1}^{(0)}\right)$.

Theorem 2. (6). Let the denominators of $a^{(v)}(V=0,1, \cdots)$ be rationalized, that is,

$$
\begin{aligned}
& a_{i}^{(v)}=\frac{C_{i, 1}^{(v)}+C_{i, 2}^{(v)} \omega+\cdots+C_{i, n}^{(v)} \omega^{n-1}}{M_{v}} \\
& i=1, \cdots, n-1 ; v=0,1, \cdots ; C_{i, j}^{(v)} \in Z \quad(j=1, \cdots, n) \\
& M_{v} \in N ; \quad Q(\omega)=Q\left(a_{1}^{(0)}, \cdots, a_{n-1}^{(0)}\right) .
\end{aligned}
$$

If there exists $a v \geqq 1$ such that

$$
M_{v}=1
$$

and if the $a_{t}^{(v)}(i=1, \cdots, n-1)$ are algebraic integers, then

$$
e=A_{0}^{(v)}+a_{1}^{(v)}(\omega) A_{0}^{(v+1)}+\cdots+a_{n-1}^{(v)}(\omega) A_{0}^{(v+n-1)}
$$

is a unit in $Q(\omega)$. The $A_{0}^{())}$are calculated by the recurrence formula

$$
\begin{gathered}
A_{0}^{(0)}=1 ; \quad A_{0}^{(1)}=A_{0}^{(2)}=\cdots=A_{0}^{(n-1)}=0, \\
A_{0}^{(n+k)}=A_{0}^{(k)}+b_{1}^{(k)} A_{0}^{(k+1)}+b_{2}^{(k)} A_{0}^{(k+2)}+\cdots \\
+b_{n-1}^{(k)} A_{0}^{(k+n-1)} \quad(k=0,1, \cdots) .
\end{gathered}
$$

2. A new periodic Jacobi-Perron algorithm. Let

$$
m=a^{6}+3 a^{3}+3, \quad \omega^{3}=m
$$


and

$$
a^{(0)}(\omega)=\left(\omega, \omega^{2}\right), \quad a \in N, \quad a \geqq 2 .
$$

It seems that the first step is to give a sufficiently good approximation for $\omega$. Since

$$
a \geqq 2, \quad a^{6}>3 a^{3}+3,
$$

we can rewrite

$$
\omega=\left(a^{6}+3 a^{3}+3\right)^{1 / 3}=a^{2}\left(1+\frac{3\left(a^{3}+1\right)}{a^{6}}\right)^{1 / 3}
$$

as

$$
\omega=a^{2}\left(1+\frac{a^{3}+1}{a^{6}}-\frac{\left(a^{3}+1\right)^{2}}{a^{12}}+\cdots\right)=a^{2}\left(1+\frac{1}{a^{3}}-\frac{\left(2 a^{3}+1\right)}{a^{12}}+\cdots\right)
$$

and we have approximately

$$
\omega=a^{2}+\frac{1}{a}-\frac{\left(2 a^{3}+1\right)}{a^{10}} .
$$

Now, since $0<1 / a-\left(2 a^{3}+1\right) / a^{10}<1$, we obtain

$$
[\omega]=a^{2} .
$$

In the following calculations, we shall use as the approximation for $\omega$,

$$
\omega=a^{2}+\frac{1}{a}
$$

since the remainder is comparatively very small. It should be noted that $a^{2}+1 / a>\omega$. We further obtain the approximation of $\omega^{2}$ :

$$
\omega^{2}=a^{4}\left(1+\frac{2\left(a^{3}+1\right)}{a^{6}}-\frac{\left(a^{3}+1\right)^{2}}{a^{12}}+\cdots\right),
$$

which is approximately

$$
\begin{aligned}
& \omega^{2}=a^{4}\left(1+\frac{2}{a^{3}}+\frac{a^{6}-2 a^{3}-1}{a^{12}}\right), \\
& \omega^{2}=a^{4}+2 a+\frac{a^{6}-2 a^{3}-1}{a^{8}} .
\end{aligned}
$$


Since $0<\left(a^{6}-2 a^{3}-1\right) / a^{8}<1$, we obtain

$$
\left[\omega^{2}\right]=a^{4}+2 a .
$$

We shall cautiously use the following approximation

$$
\omega^{2}=a^{4}+2 a+1 / a^{2}
$$

keeping in mind that $a^{4}+2 a+1 / a^{2}>\omega^{2}$. We now have the beginning of the JPA for

$$
\begin{aligned}
& a^{(0)}=\left(\omega, \omega^{2}\right), \\
& b^{(0)}=\left(a^{2}, a^{4}+2 a\right),
\end{aligned}
$$

and obtain, by definition,

$$
a^{(1)}=\left(\frac{\omega^{2}-\left(a^{4}+2 a\right)}{\omega-a^{2}}, \frac{1}{\omega-a^{2}}\right) .
$$

We can now obtain the rationalization of the denominator directly, keeping in mind that

$(2.10) \omega^{3}-a^{6}=3\left(a^{3}+1\right)$,

$$
\begin{aligned}
a^{(1)}(\omega) & =\left(\frac{\left(\omega^{2}-\left(a^{4}+2 a\right)\right)\left(\omega^{2}+a^{2} \omega+a^{4}\right)}{\left(\omega-a^{2}\right)\left(\omega^{2}+a^{2} \omega+a^{4}\right)}, \frac{\left(\omega^{2}+a^{2} \omega+a^{4}\right)}{\left(\omega-a^{2}\right)\left(\omega^{2}+a^{2} \omega+a^{4}\right)}\right), \\
& =\left(\frac{-2 a \omega^{2}+\left(a^{3}+3\right) \omega+a^{2}\left(a^{3}+3\right)}{\omega^{3}-a^{6}}, \frac{\omega^{2}+a^{2} \omega+a^{4}}{\omega^{3}-a^{6}}\right),
\end{aligned}
$$

(2.11) $a^{(1)}(\omega)=\left(\frac{-2 a \omega^{2}+\left(a^{3}+3\right) \omega+a^{2}\left(a^{3}+3\right)}{3\left(a^{3}+1\right)}, \frac{\omega^{2}+a^{2} \omega+a^{4}}{3\left(a^{3}+1\right)}\right)$.

Now, using the approximation formulas (2.5) and (2.8), we can write $-2 a \omega^{2}+\left(a^{3}+3\right) \omega+a^{2}\left(a^{3}+3\right)$ as

$$
-2 a\left(a^{4}+2 a+\frac{1}{a^{2}}\right)+\left(a^{3}+3\right)\left(a^{2}+\frac{1}{a}\right)+a^{5}+3 a^{2}=2 a^{2}+a+\frac{1}{a} .
$$

Therefore,

$$
b_{1}^{(1)}=\left[\frac{2 a^{2}+a+\frac{1}{a}}{3\left(a^{3}+1\right)}\right]=0 .
$$

We further obtain, 


$$
\omega^{2}+a^{2}+a^{4}=a^{4}+2 a+\frac{1}{a^{2}}+a^{4}+a+a^{4}=3 a^{4}+3 a+\frac{1}{a^{2}} .
$$

Hence

$$
b_{2}^{(1)}=\left[\frac{3 a\left(a^{3}+1\right)+\frac{1}{a^{2}}}{3\left(a^{3}+1\right)}\right]=a .
$$

We write for the sake of convenience, as will also be done in the sequel, $a^{(1)}(\omega)$ and $b^{(1)}$.

$$
\begin{aligned}
& a^{(1)}(\omega)=\left(\frac{-2 a \omega^{2}+\omega\left(a^{3}+3\right)+a^{2}\left(a^{3}+3\right)}{3\left(a^{3}+1\right)}, \frac{\omega^{2}+a^{2} \omega+a^{4}}{3\left(a^{3}+1\right)}\right), \\
& b^{(1)}=(0, a) .
\end{aligned}
$$

We obtain the next vector, by definition,

$$
\begin{aligned}
a^{(2)}(\omega)=\left(\frac{\omega^{2}+a^{2} \omega-\left(2 a^{4}+3 a\right)}{-2 a \omega^{2}+\left(a^{3}+3\right) \omega+a^{2}\left(a^{3}+3\right)},\right. \\
\left.\frac{3\left(a^{3}+1\right)}{-2 a \omega^{2}+\left(a^{3}+3\right) \omega+a^{2}\left(a^{3}+3\right)}\right)
\end{aligned}
$$

$$
\begin{aligned}
a^{(2)}(\omega)=\left(\frac{-a \omega^{2}+\left(2 a^{3}+3\right) \omega-a^{2}\left(a^{3}+2\right)}{3 a^{6}+10 a^{3}+9},\right. \\
\left.\frac{\left(a^{3}+3\right) \omega^{2}+a^{2}\left(a^{3}+1\right) \omega+a\left(a^{3}+2\right)\left(a^{3}+3\right)}{3 a^{6}+10 a^{3}+9}\right) .
\end{aligned}
$$

For the calculation of $b_{1}^{(2)}$ and $b_{2}^{(2)}$, we obtain

$$
\begin{aligned}
-a \omega^{2}+\left(2 a^{3}+3\right) & \omega-a^{2}\left(a^{3}+2\right) \\
= & -a\left(a^{4}+2 a+\frac{1}{a^{2}}\right)+\left(2 a^{3}+3\right)\left(a^{2}+\frac{1}{a}\right)-a^{2}\left(a^{3}+2\right),
\end{aligned}
$$

which simplifies to $a^{2}+2 / a$. Hence

$$
b_{1}^{(2)}=\left[\frac{a^{2}+\frac{2}{a}}{3 a^{6}+10 a^{3}+9}\right]=0 .
$$

Now

$$
\begin{aligned}
& \left(a^{3}+3\right) \omega^{2}+a^{2}\left(a^{3}+1\right) \omega+a\left(a^{3}+2\right)\left(a^{3}+3\right) \\
& \quad=\left(a^{3}+3\right)\left(a^{4}+2 a+\frac{1}{a^{2}}\right)+\left(a^{5}+a^{2}\right)\left(a^{2}+\frac{1}{a}\right)+a^{7}+5 a^{4}+6 a \\
& \quad=3 a^{7}+12 a^{4}+14 a+\frac{3}{a^{2}} .
\end{aligned}
$$


Therefore,

$$
\begin{aligned}
& b_{2}^{(2)}=\left[\frac{3 a^{7}+10 a^{4}+9 a}{3 a^{6}+10 a^{3}+9}+\frac{2 a^{4}+5 a+\frac{3}{a^{2}}}{3 a^{6}+10 a^{3}+9}\right] . \\
& b_{2}^{(2)}=a .
\end{aligned}
$$

Now,

$$
\begin{aligned}
& a^{(2)}(\omega)=\left(\frac{-a \omega^{2}+\left(2 a^{3}+3\right) \omega-a^{2}\left(a^{3}+2\right)}{3 a^{6}+10 a^{3}+9},\right. \\
& \left.\frac{\left(a^{3}+3\right) \omega^{2}+a^{2}\left(a^{3}+1\right) \omega+a\left(a^{3}+2\right)\left(a^{3}+3\right)}{3 a^{6}+10 a^{3}+9}\right), \\
& b^{(2)}=(0, a) .
\end{aligned}
$$

Therefore,

$$
\begin{aligned}
& \text { (2.17) } a^{(3)}(\omega)=\left(\frac{\left(a^{3}+3\right) \omega^{2}+a^{2}\left(a^{3}+1\right) \omega-a\left(2 a^{6}+5 a^{3}+3\right)}{-a \omega^{2}+\left(2 a^{3}+3\right) \omega-a^{2}\left(a^{3}+2\right)},\right. \\
& \left.\frac{3 a^{6}+10 a^{3}+9}{-a \omega^{2}+\left(2 a^{3}+3\right) \omega-a^{2}\left(a^{3}+2\right)}\right) \\
& \text { (2.18) } a^{(3)}(\omega)=\left(\omega+a^{2}, \omega^{2}+a^{2} \omega+a\left(a^{3}+1\right)\right) .
\end{aligned}
$$

The reader can now verify the continuation of the algorithm and prove

$$
a^{(7)}(\omega)=a^{(4)}(\omega)
$$

This important result can now be expressed in the following theorem:

THEOREM 2.1. Let $m$ be a cube-free natural number of the form $m=a^{6}+3 a^{3}+3$, where $a$ is a natural number greater than or equal to 2. Let $\omega^{3}=m$. Then the JPA of the vector $a^{(0)}(\omega)=\left(\omega, \omega^{2}\right)$ is periodic. The length of the primitive preperiod is four and has the form

$$
\begin{aligned}
a^{(0)}(\omega)= & \left(\omega, \omega^{2}\right), \\
a^{(1)}(\omega)= & \left(\frac{-2 a \omega^{2}+\left(a^{3}+3\right) \omega+a^{2}\left(a^{3}+3\right)}{3\left(a^{3}+1\right)}, \frac{\omega^{2}+a^{2} \omega+a^{4}}{3\left(a^{3}+1\right)}\right), \\
a^{(2)}(\omega)= & \left(\frac{-a \omega^{2}+\left(2 a^{3}+3\right) \omega-a^{2}\left(a^{3}+2\right)}{3 a^{6}+10 a^{3}+9},\right. \\
\left.\frac{\left(a^{3}+3\right) \omega^{2}+a^{2}\left(a^{3}+1\right) \omega+a\left(a^{3}+2\right)\left(a^{3}+3\right)}{3 a^{6}+10 a^{3}+9}\right), & \\
a^{(3)}(\omega)= & \left(\omega+a^{2}, \omega^{2}+a^{2} \omega+a\left(a^{3}+1\right)\right) \\
b^{(3)}= & \left(2 a^{2}, 3 a^{4}+4 a\right) .
\end{aligned}
$$


The period of the JPA of $a^{(0)}(\omega)$ has length three and is of the form

$$
\begin{aligned}
a^{(4)}(\omega) & =\left(\frac{-3 a \omega^{2}+3 \omega+3 a^{2}\left(a^{3}+2\right)}{3\left(a^{3}+1\right)}, \frac{\omega^{2}+a^{2} \omega+a^{4}}{3\left(a^{3}+1\right)}\right), \\
b^{(4)} & =(0, a), \\
a^{(5)}(\omega) & =\left(\frac{\omega-a^{2}}{3\left(a^{3}+1\right)}, \frac{\omega^{2}+a^{2} \omega+a\left(a^{3}+3\right)}{3\left(a^{3}+1\right)}\right), \\
b^{(5)} & =(0, a), \\
a^{(6)}(\omega) & =\left(\omega+2 a^{2}, \omega^{2}+a^{2} \omega+a\left(a^{3}+3\right)\right) \\
b^{(6)} & =\left(3 a^{2}, 3 a^{3},+3 a\right) .
\end{aligned}
$$

In the above theorem, we have excluded $a=1$ and this is done because, if $a=1$, then $m=7=2^{3}-1=D^{3}-1, D=2$ and this form appears to be a special case of Bernstein's periodic Jacobi-Perron algorithm as stated in Theorem 3.3, where $m=D^{3}-d, d \mid D$. Here $D=2, d=1$. But his form is not exactly a special case because $D \geqq 2 d(n-1)$ is not satisfied. Yet, the JPA of $a^{(0)}=\left(\sqrt[3]{7}, \sqrt[3]{7^{2}}\right)$ is periodic and one obtains the following:

$$
\begin{aligned}
a^{(0)}(\omega) & =\left(\omega, \omega^{2}\right) ; \quad \omega=\sqrt[3]{7}, \\
b^{(0)} & =(1,3), \\
a^{(1)}(\omega) & =\left(\frac{-2 \omega^{2}+4 \omega+4}{6}, \frac{\omega^{2}+\omega+1}{6}\right), \\
b^{(1)} & =(0,1), \\
a^{(2)}(\omega) & =\left(\frac{-\omega^{2}+5 \omega-3}{22}, \frac{4 \omega^{2}+2 \omega+12}{22}\right), \\
b^{(2)} & =(0,1), \\
a^{(3)}(\omega) & =\left(\omega+1, \omega^{2}+\omega+2\right), \\
b^{(3)} & =(2,7), \\
a^{(4)}(\omega) & =\left(\frac{-3 \omega^{2}+3 \omega+9}{6}, \frac{\omega^{2}+\omega+1}{6}\right), \\
b^{(4)} & =(0,1), \\
a^{(5)}(\omega) & =\left(\frac{\omega-1}{6}, \frac{\omega^{2}+\omega+4}{6}\right), \\
b^{(5)} & =(0,1), \\
a^{(6)}(\omega) & =\left(\omega+2, \omega^{2}+\omega+1\right), \\
b^{(7)} & =(3,6), \\
a^{(7)}(\omega) & =a^{(4)}(\omega) .
\end{aligned}
$$


Comparing the above formulas with values obtained for $a^{(v)}(\omega)$, $(v=1, \cdots, 6)$ in Theorem 2.1, we immediately see that Theorem 2.1 also holds for the case where $a=1$.

We have yet to show that there are infinitely many cubic fields $Q(\omega), \omega^{3}=a^{6}+3 a^{3}+3$ or that the equation $a^{6}+3 a^{3}+3=t y^{3}$, where $t$ is a fixed number, $a$ and $y$ are indeterminants, has only a finite number of solutions. We obtain, multiplying by $a^{3}$,

$$
\left(a^{3}+1\right)^{3}-1=t(y a)^{3}
$$

denoting $a^{3}+1=x, y a=z$, we obtain

$$
x^{3}-t z^{3}=1
$$

and this Diophantine equation, by a famous theorem of Nagell [10], has at most one nontrivial solution $\left(x_{1}, z_{1}\right)$.

3. Units in $Q(\omega), \omega^{3}=m=a^{6}+3 a^{3}+3$. In this chapter, we will calculate units in $Q(\omega)$. Since $M_{3}=1$, we can calculate a unit using the results in Theorem 2. Also, since the JPA of $a^{(0)}(\omega)=\left(\omega, \omega^{2}\right)$ is periodic, another unit can be calculated with the help of Theorem 1.

Using formula (1.8) and noting that $n=3$ and $V=3$, we obtain

$$
e=A_{0}^{(3)}+a_{1}^{(3)} A_{0}^{(4)}+a_{2}^{(3)} A_{0}^{(5)},
$$

and from formula (2.18),

$$
e=A_{0}^{(3)}+\left(\omega+a^{2}\right) A_{0}^{(4)}+\left(\omega^{2}+a^{2} \omega+a^{4}+a\right) A_{0}^{(5)} .
$$

In order to calculate $A_{0}^{(3)}, A_{0}^{(4)}$ and $A_{0}^{(5)}$, we need $b^{(1)}$ and $b^{(2)}$ which are, according to formulas (2.12), (2.13), (2.15), and (2.16),

$$
\begin{aligned}
& b^{(1)}=(0, a), \\
& b^{(2)}=(0, a) .
\end{aligned}
$$

Using formula (1.9), we obtain

$$
\begin{aligned}
& A_{0}^{(3)}=A_{0}^{(0)}+b_{1}^{(1)} A_{0}^{(1)}+b_{2}^{(0)} A_{0}^{(2)}=1+b_{1}^{(0)} \cdot 0+b_{2}^{(0)} \cdot 0=1, \\
& A_{0}^{(4)}=A_{0}^{(1)}+b_{1}^{(1)} A_{0}^{(2)}+b_{2}^{(1)} A_{0}^{(3)}=0+b_{1}^{(1)} \cdot 0+a \cdot 1=a, \\
& A_{0}^{(5)}=A_{0}^{(2)}+b_{1}^{(2)} A_{0}^{(3)}+b_{2}^{(2)} A_{0}^{(4)}=0+0 \cdot 1+a \cdot a=a^{2} ; \\
& A_{0}^{(3)}=1, \quad A_{0}^{(4)}=a, \quad A_{0}^{(5)}=a^{2} .
\end{aligned}
$$


Now, from (3.2) and (3.4),

$$
\begin{aligned}
e & =1+\left(\omega+a^{2}\right) a\left(\omega^{2}+a^{2} \omega+\left(a^{4}+a\right)\right) a^{2} \\
& =a^{2} \omega^{2}+\left(a^{4}+a\right) \omega+a^{6}+2 a^{3}+1, \\
e & =\left(1+a^{3}\right)^{2}+a\left(1+a^{3}\right) \omega+a^{2} \omega^{2} .
\end{aligned}
$$

This is a comparatively simple form for $e$. We shall now calculate $e^{-1}$ as follows.

$$
\begin{aligned}
e^{-1} & =\frac{1}{\left(1+a^{3}\right)^{2}+a\left(1+a^{3}\right) \omega+a^{2} \omega^{2}}, \\
& =\frac{1+a^{3}-a \omega}{\left(\left(1+a^{3}\right)^{2}+a\left(1+a^{3}\right) \omega+a^{2} \omega^{2}\right)\left(1+a^{3}-a \omega\right)}, \\
& =\frac{1+a^{3}-a \omega}{\left(1+a^{3}\right)^{3}-a^{3} \omega^{3}}=\frac{1+a^{3}-a \omega}{a^{9}+3 a^{6}+3 a^{3}+1-a^{9}-3 a^{6}-3 a^{3}}, \\
e^{-1} & =1+a^{3}-a \omega
\end{aligned}
$$

which is indeed an elegant and a beautiful expression for a unit in $Q(\omega)$.

Since the JPA of $\left(\omega, \omega^{2}\right)$ is also periodic, formula (1.5), in view of Theorem 2, becomes

$$
e_{1}=\prod_{i=4}^{6} a_{2}^{(i)}=a_{2}^{(4)} a_{2}^{(5)} a_{2}^{(6)}
$$

We obtain

$$
\begin{aligned}
a_{2}^{(4)} a_{2}^{(5)} a_{2}^{(6)} & =\frac{1}{\omega-a^{2}} \cdot \frac{3\left(a^{3}+1\right)}{-3 a \omega^{2}+3 \omega+3 a^{2}\left(a^{3}+2\right)} \cdot\left(\omega^{2}+a^{2} \omega+a^{4}\right) \\
& =\frac{3\left(a^{3}+1\right)\left(\omega^{2}+a^{2} \omega+a^{4}\right)}{\left(\omega-a^{2}\right)\left(-3 a \omega^{2}+3 \omega+3 a^{2}\left(a^{3}+2\right)\right)} \\
& =\frac{3\left(a^{3}+1\right)\left(\omega^{2}+a^{2} \omega+a^{4}\right)}{3\left(a^{3}+1\right)\left(\omega^{2}+a^{2} \omega-\left(2 a^{4}+3 a\right)\right)} \\
& =\frac{\left(\omega^{2}+a^{2} \omega+a^{4}\right)\left(\omega-a^{2}\right)}{\left.\omega^{2}+a^{2} \omega-\left(2 a^{4}+3 a\right)\right)\left(\omega-a^{2}\right)} \\
& =\frac{3\left(a^{3}+1\right)}{-3 a\left(a^{3}+1\right) \omega+3\left(a^{3}+1\right)^{2}} \\
& =\frac{1}{-a \omega+\left(a^{3}+1\right)}, \\
e_{1} & =a_{2}^{(4)} a_{2}^{(5)} a_{2}^{(6)}=\frac{1}{a^{3}+1-a \omega} .
\end{aligned}
$$


Now, taking $e_{1}^{-1}$, we obtain

$$
e^{-1}=a^{3}+1-a \omega
$$

which is exactly identical to the unit given in (3.6). The question of the fundamentality of this unit is yet to be answered. We shall do that in the following chapter.

4. The fundamentality of units in $Q(\omega), \omega^{3}=m=$ $a^{6}+3 a^{3}+3$. In the preceding chapter, we showed that $e=1+a^{3}-a \omega$ is a unit in $Q(\omega), \omega^{3}=m=a^{6}+3 a^{3}+3$. This unit provides a nontrivial solution of the famous Nagell equation

$$
x^{3}-m y^{3}=1, \quad \omega^{3}=m=a^{6}+3 a^{3}+3 .
$$

To see this, one simply sets $x=1+a^{3}, y=a$. Although Nagell could not prove whether or not $x^{3}-m y^{3}=1$ has a solution, he did prove the following important theorem.

THEOREM 3. If $\left(x_{1}, y_{1}\right)$ is a nontrivial solution of $x^{3}-m y^{3}=1$, when $m$ is a cube-free rational integer, then $x_{1}-y \omega, \omega^{3}=m$ is a fundamental unit of $Q(\omega)$, or the square of a fundamental unit. The latter happens only when $m=19,20$, or 28 .

In view of Nagell's theorem, we only need to check to see if there exists an $a \in N$ such that $a^{6}+3 a^{3}+3=19,20$, or 28 . However, it is obvious that no such $a$ exists and therefore we have the following important result.

THEOREM 4.2. In the field $Q(\omega)$, where $\omega^{3}=a^{6}+3 a^{3}+3$ and $a$ is $a$ natural number, $e=1+a^{3}-a \omega$ is a fundamental unit.

\section{REFERENCES}

1. L. Bernstein, Periodic continued fractions of degree $n$ by Jacobi's algorithm, J. F. D. Reine Angew. Math., 213 (1964), 31-38.

2. - Periodicity of Jacobi's algorithm for a special type of cubic irrationals, J. F. D. Reine Angew. Math., 213 (1964), 134-146.

3. - Periodische Jacobische Algorithmen, Archiv Der Mathematik, 15 (1964), 421-429.

4. - Representation of $\left(D^{n}-d\right)^{1 / n}$ as a periodic continued fraction by Jacobi's algorithm, Math. Nachrichten, 109 (1965), 179-200.

$5 .+$ New infinite classes of periodic Jacobi-Perron algorithms, Pacific J. Math., 16 (1965), $1-31$.

6. - A 3-Dimensional Periodic Jacobi-Perron Algorithm of Period Length 8, J. Number Theory, 4 (1972), 48-69.

7. Lecture Notes in Mathematics 207; The Jacobi-Perron Algorithm: Its Theory and Application. Springer-Verlag, Berlin, 1971. 
8. L. Bernstein and H. Hasse, Einheitenberechnung Mittels des Jacobi-Perronschen Algorithmus, J.

F. D. Reine Angew. Math., 218, 165, pp. 51-69.

9. C. G. J. Jacobi, Allgemeine Theorie der Kettenbruchaehnlichen Algorithmen etc. J. F. D. Reine Angew. Math., 69 (1869), 29-64.

10. M. T. Nagell, Einige Gleichungen von der Form $a y^{2}+b y^{2}+C$, Arch. Norske Vid, Akad. Oslo, 7 (1930), 1-15.

11. O. Perron, Grundlagen für eine Theorie des Jacobischen Kettenbruch Algorithmus, Math. Annalen, 64k (1907), 1-76.

Received November 20, 1975 and in revised form March 25, 1976. I am extremely grateful to the referee for suggestions that contributed substantially to the clarity and compactness of this paper.

Science Research Associates, Inc., Chicago 




\section{Pacific Journal of Mathematics}

Vol. 64, No. 1

May, 1976

Walter Allegretto, Nonoscillation theory of elliptic equations of order $2 n \ldots \ldots \quad 1$

Bruce Allem Anderson, Sequencings and starters.................. 17

Friedrich-Wilhelm Bauer, A shape theory with singular homology .......... 25

John Kelly Beem, Characterizing Finsler spaces which are

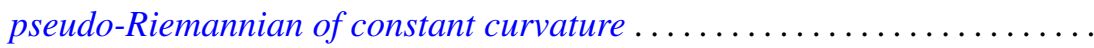

Dennis K. Burke and Ernest A. Michael, On certain point-countable

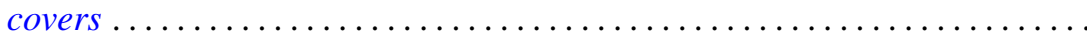

Robert Chen, A generalization of a theorem of Chacon ............... 93

Francis H. Clarke, On the inverse function theorem ................ 97

James Bryan Collier, The dual of a space with the Radon-Nikodým

property ....................................... 103

John E. Cruthirds, Infinite Galois theory for commutative rings ............ 107

Artatrana Dash, Joint essential spectra......................... 119

Robert M. DeVos, Subsequences and rearrangements of sequences in FK

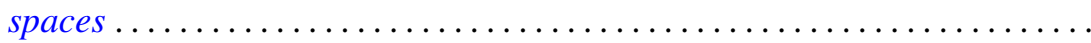

Geoffrey Fox and Pedro Morales, Non-Hausdorff multifunction generalization

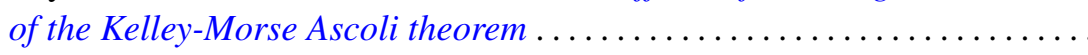

Richard Joseph Fleming, Jerome A. Goldstein and James E. Jamison, One

parameter groups of isometries on certain Banach spaces.............

Robert David Gulliver, II, Finiteness of the ramified set for branched

immersions of surfaces

Kenneth Hardy and István Juhász, Normality and the weak cb property ...... 167

C. A. Hayes, Derivation of the integrals of $L^{(q)}$-functions.

Frederic Timothy Howard, Roots of the Euler polynomials .

Robert Edward Jamison, II, Richard O'Brien and Peter Drummond Taylor, On

embedding a compact convex set into a locally convex topological vector space ....................................

Andrew Lelek, An example of a simple triod with surjective span smaller than span ...

Janet E. Mills, Certain congruences on orthodox semigroups

Donald J. Newman and A. R. Reddy, Rational approximation of $e^{-x}$ on the positive real axis.

John Robert Quine, Jr., Homotopies and intersection sequences ...

Nambury Sitarama Raju, Periodic Jacobi-Perron algorithms and fundamental units ....

Herbert Silverman, Convexity theorems for subclasses of univalent functions. . .

Charles Frederick Wells, Centralizers of transitive semigroup actions and endomorphisms of trees.........................

Volker Wrobel, Spectral approximation theorems in locally convex spaces ..................... 